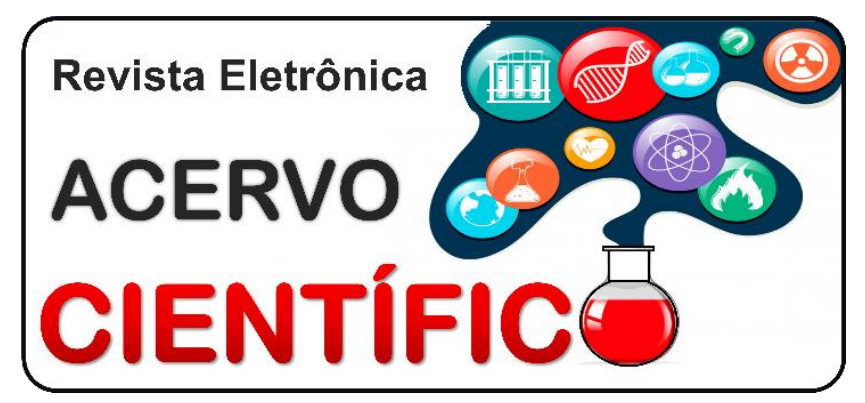

ARTIGO ORIGINAL

Recebido em: 3/2020

Aceito em: 4/2020

Publicado em: 5/2020

\title{
Infecção do trato urinário em pacientes idosos em atendimento domiciliar: prevalência, manifestações clínicas e tratamento
}

\author{
Infection of urinary tract in the elderly in home care: prevalence, clinical manifestations and \\ treatment
}

Infección del tracto urinario em ancianos em condición de tratamiento domiciliar: prevalencia, manifestaciones clínicas y tratamiento

Lorena Resende e Silva ${ }^{1}$, Priscilla Baltazar Domingos ${ }^{1}$, Thaís Corrêa Nascimento ${ }^{*}$, Lucas Fernandes Macedo', Rodrigo Tavares Silva1.

Resumo: Descrever e analisar a epidemiologia das manifestações clínicas e terapia medicamentosa da infecção do trato urinário (ITU) em pacientes idosos em atendimento domiciliar. Trata-se de um estudo retrospectivo e transversal, de análise de prontuários de idosos atendidos em domicílio, provenientes de serviço de saúde suplementar. Foram elegíveis para o estudo 59 pacientes, com idade média de $84,4 \pm 9$ anos; predomínio do gênero feminino (83,1\%), hipertensos $(49,1 \%)$, cardiopatas $(42,3 \%)$ e diabéticos $(23,7 \%)$. Os agentes etiológicos mais frequentes foram Escherichia coli $(n=66)$ e Klebsiella sp $(n=32)$. A partir de análise bivariada, houve relação positiva $(p<0,05)$ entre: idade com alteração das características da urina $(p=0,03)$; ITU de repetição com doença de Alzheimer $(p=0,01)$ e restrição ao leito com doença de Parkinson $(p=0,03)$. Não houve relação significativa entre ITU e diabetes. Houve predomínio de ITU no sexo feminino. Observouse que as alterações nas características da urina e no estado mental foram os sintomas mais prevalentes. Houve relação gradativa entre o avançar da idade com alteração nas características da urina e a recorrência de ITU. Verificou-se que a imobilidade, leva à deterioração progressiva de vários sistemas, principalmente o urinário. O tratamento incialmente é empírico e, posteriormente, baseia-se no antibiograma e cultura.

Palavras-chave: Sistema urinário, Saúde do idoso, Bacteriúria.

Abstract: To describe and analyze the epidemiology of clinical manifestations and drug therapy of UTI in elderly patients in home care. It's a retrospective, cross-sectional study, based on analysis of medical records of elderly people home assisted, from supplementary health service. 59 patients, with mean age of $84 \pm 9$ year, were eligible for the study: female sex (83.1\%), hypertension $(49.1 \%)$, heart disease $(42.3 \%)$ and diabetes $(23.7 \%)$. The most frequent etiological agents were Escherichia coli $(n=66)$ and Klebsiella sp $(n=$ 32). From bivariate analysis, there was positive relation $(p<0.05)$ between: age with altered urine characteristics $(p=0.03)$; recurrent ITU with Alzheimer's disease $(p=0.01)$ and bed restriction with Parkinson's disease $(p=0.03)$. There was no significant relation between UTI and diabetes. There was predominance of UTI in the female sex. Changes in urine characteristics and mental state were the most prevalent symptoms.

${ }^{1}$ Universidade de Franca (UNIFRAN). Franca - SP. *E-mail: thata_cn@hotmail.com 
There was gradual relation between the progression of age with modifications in urine characteristics and recurrence. Immobility has been found to lead to progressive deterioration of several systems, especially the urinary system. The treatment is initially empirical and should be adjusted in accordance with antibiogram and culture results.

Keywords: Urinary tract, Health of the elderly, Bacteriuria.

Resumen: Describir y analizar la epidemiología de las manifestaciones clínicas y el tratamiento farmacológico para la infección del tracto urinario (ITU) en pacientes de edad avanzada en atención domiciliaria. Este es un estudio retrospectivo y transversal, que analiza los registros médicos de las personas mayores atendidas en el hogar, desde un servicio de salud complementario. 59 pacientes fueron elegibles para el estudio, con una edad media de 84,4 \pm 9 años; predominio de mujeres (83.1\%), hipertensos (49.1\%), pacientes cardíacos $(42.3 \%)$ y diabéticos $(23.7 \%)$. Los agentes etiológicos más frecuentes fueron Escherichia coli $(n=66)$ y Klebsiella sp $(n=32)$. De un análisis bivariado, hubo una relación positiva $(p<0.05)$ entre: edad con cambios en las características de la orina $(p=0.03)$; ITU recurrente con enfermedad de Alzheimer $(p=0.01)$ y restricción de cama con enfermedad de Parkinson $(p=0.03)$. No hubo una relación significativa entre la infección urinaria y la diabetes. Hubo un predominio de ITU en las mujeres. Se observó que los cambios en las características de la orina y el estado mental eran los síntomas más frecuentes. Hubo una relación gradual entre la edad avanzada con cambios en las características de la orina y la recurrencia de la infección urinaria. Se encontró que la inmovilidad conduce al deterioro progresivo de varios sistemas, principalmente el sistema orinario. El tratamiento es inicialmente empírico y, luego, se basa en el antibiograma y el cultivo.

Palabras-clave: Sistema urinario, Salud del anciano, Bacteriuria.

\section{INTRODUÇÃO}

No Brasil, é definida como idosa a pessoa que tem 60 anos ou mais de idade. O envelhecimento da população brasileira irá impactar os serviços de saúde, visto que, a cada ano, 650 mil novos indivíduos passam a ser considerados idosos, e grande parte destes apresentam doenças crônicas e alguma limitação funcional. $O$ trato urinário é um dos locais mais comuns de infecções bacterianas em populações idosas. (VERAS R, 2009).

A Infecção do Trato Urinário (ITU) é definida como a colonização microbiana na urina. Nas ITU adquiridas na comunidade, os agentes etiológicos mais prevalentes, por ordem de frequência, são: Escherichia coli, espécies de Proteus e de Klebsiella. O patógeno E. coli causa de $70 \%$ a $85 \%$ das infecções adquiridas na comunidade, e $50 \%$ a $60 \%$ em pacientes idosos institucionalizados. Além disso, quanto maior for o grau de dependência e debilidade do idoso, maior é a prevalência de ITU. Considera-se que um paciente seja portador de ITU de repetição quando este é acometido por 3 ou mais episódios de ITU no período de um ano. A mortalidade diretamente atribuída por ITU é incomum, mesmo na população idosa. (BEVERIDGE LA, et al., 2011; CORRÊA EF e MONTALVÃO ER, 2010).

O aumento da prevalência em ambos os sexos ocorre pela diminuição dos mecanismos de defesa (imunossenescência) como, por exemplo, a alteração na liberação de imunoglobulinas locais. Outros fatores que predispõem os idosos a maior risco de desenvolver infecções do trato urinário são a institucionalização e o uso crônico de antibióticos, favorecendo a resistência bacteriana, além da presença de comorbidades que aumentam a susceptibilidade às infecções $A$ ocorrência é maior no sexo feminino, embora ocorra também aumento no sexo masculino após os 50 anos, devido às características anatômicas e às alterações fisiológicas do aparelho geniturinário. (BEVERIDGE LA, et al., 2011; CORRÊA EF e MONTALVÃO ER, 2010; RORIZ-FILHO JS, et al., 2010)

A incidência de episódios de bacteriúria assintomática em idosos da comunidade é notável, acomete $20 \%$ das mulheres com mais de 80 anos e tende a aumentar com o avanço da idade. Já em idosos institucionalizados a prevalência de bacteriúria assintomática é ainda maior. Os fatores determinantes da alta 
frequência de bacteriúria são principalmente múltiplas comorbidades crônicas, como doenças cardiovasculares, diabetes mellitus (DM), hipertrofia prostática nos homens, comuns nesta população. Idosos institucionalizados, portadores de doenças neurológicas crônicas como síndromes demenciais, Alzheimer e Parkinson, com déficit de funcionalidade, diminuição cognitiva e incontinência urinária e fecal, frequentemente possuem bacteriúria assintomática associada (BEVERIDGE LA, et al., 2011; CORRÊA EF e MONTALVÃO ER, 2010).

O quadro clínico clássico da ITU é manifestado por disúria, polaciúria, urgência miccional, incontinência urinária e noctúria. Em pacientes idosos, principalmente naqueles com déficit cognitivo que prejudique a comunicação, podem estar mascarados ou ausentes, retardando o diagnóstico. (BEVERIDGE LA, et al., 2011; CORRÊA EF e MONTALVÃO ER, 2010).

O tratamento da ITU é empírico, de acordo com a epidemiologia local. Para ITU baixa (cistite) de origem comunitária em jovens imunocompetentes e mulheres recomenda-se o uso de Norfloxacino $400 \mathrm{mg}$ via oral de 12/12h por 7 dias ou Nitrofurantoína $100 \mathrm{mg}$ via oral de $616 \mathrm{~h}$ por 7 dias, ou ainda Cefalexina $500 \mathrm{mg}$ por via oral de 6/6h por 7 dias. Se necessário, a terapia deve ser ajustada em conformidade com os resultados do antibiograma e cultura, como por exemplo, recorrer ao descalonamento e/ou usar esquemas terapêuticos mais custo efetivos. (CORRÊA EF e MONTALVÃO ER, 2010; RORIZ-FILHO JS, et al., 2010; GUPTA K, et al, 2011).

Entre os pacientes com ITU de repetição, observa-se aumento na incidência de microorganismos resistentes aos antimicrobianos mais utilizados, dificultando o seu tratamento. Devido a isso, exige-se a utilização de antibióticos de largo espectro com frequência cada vez maior, o que também incentiva a pesquisa de novos métodos de tratamento $\mathrm{A}$ antibioticoterapia profilática ainda não possui consenso na literatura. Por outro lado, terapias alternativas como o uso do Cranberry e estrogênio tópico se mostram eficazes na redução da ITU de repetição (RORIZ-FILHO JS, et al, 2010; MATTHEWS SJ e LANCASTER JW, 2011; FRANÇA ACYR, et al., 2014).

Em última análise, visto o aumento demográfico e fragilidade própria da população idosa, aliados ao benefício clínico do diagnóstico precoce e ausência de estudos robustos neste cenário clínico, torna-se relevante descrever e analisar a epidemiologia das manifestações clínicas, fatores de risco e terapia medicamentosa da ITU em pacientes idosos em atendimento domiciliar. (BEVERIDGE LA, et al., 2011; CORRÊA EF e MONTALVÃO ER, 2010).

\section{MÉTODOS}

Trata-se de um estudo retrospectivo, transversal, e que incluiu pacientes idosos atendidos em domicílio no período de junho de 2015 a maio de 2017, provenientes de serviço de saúde suplementar. Foram incluídos os pacientes em assistência domiciliar e residentes na cidade do serviço de saúde suplementar, com idade maior ou igual a 60 anos, e com diagnóstico de ITU.

O diagnóstico de ITU foi definido de acordo com protocolo institucional, de autoria do médico infectologista responsável, no qual havia necessidade de pelo menos uma das seguintes alterações: febre $>38^{\circ}$, disúria, urgência miccional, dor abdominal baixa/lombar, alteração do estado mental, prostração e/ou desorientação e alteração da coloração e/ou odor da urina.

Foram excluídos do estudo os pacientes em uso de cateter vesical e com exames de uroculturas negativas. Os prontuários dos pacientes incluídos no estudo foram analisados, por três dos cinco pesquisadores, entre setembro e novembro de 2017. Foram coletadas e analisadas as seguintes variáveis clínicas e laboratoriais (Quadro 1). 
Quadro 1 - Variáveis clínicas e laboratoriais analisadas no estudo.

\begin{tabular}{|l|}
\hline Idade \\
\hline Sexo \\
\hline Quadro clínico diagnóstico da ITU \\
\hline Febre $>38^{\circ} \mathrm{C}$ \\
\hline Disúria \\
\hline Alteração da característica da urina \\
\hline Alteração do nível de consciência \\
\hline Comorbidades \\
\hline Doenças cardiovasculares \\
\hline Diabetes \\
\hline Doença de Parkinson ou Alzheimer \\
\hline Síndromes demenciais \\
\hline Doenças pulmonares e renais \\
\hline Doenças neuropsiquiátrica \\
\hline Exame laboratorial \\
\hline Urocultura \\
\hline Antibiograma \\
\hline Terapia antibiótica \\
\hline Caráter da ITU \\
\hline Primo-infecção \\
\hline Recorrente \\
\hline
\end{tabular}

Fonte: Silva LR, et al., 2020.

A correlação entre a presença de ITU e as variáveis clínicas coletadas foi analisada pelos testes estatísticos Coeficiente de Correlação de Person, Coeficiente de Correlação de Spearman, Correlação pontobisserial e coeficiente Phi de Correlação, sendo considerados estatisticamente significante os valores de $p<$ 0,05. Este estudo está de acordo com a Resolução ํㅜ 466/2012 e a Resolução no 510/2016 e foi aprovado pelo Comitê de Ética em Pesquisa sob o registro CEP 73217117.6.0000.5495.

\section{RESULTADOS}

No período de junho de 2015 a maio de 2017, foram diagnosticados 138 casos de ITU em 64 pacientes idosos em atendimento domiciliar na instituição, com taxa de 2,15 casos/paciente. Foram elegíveis para este estudo 59 pacientes que apresentaram 133 episódios de ITU. Cinco pacientes foram excluídos por uso de cateter vesical e idade menor que 60 anos.

Foram elegíveis para o estudo 59 pacientes, com média de idade de $84,4 \pm 9$ anos; predomínio do gênero feminino $(83,1 \%)$ e elevada prevalência de hipertensão $(49,1 \%)$, seguido de cardiopatia $(42,3 \%)$ e diabetes (23,7\%). Nesta população foram diagnosticados 133 episódios de ITU, com taxa de 2,25 casos/paciente.

Os pacientes apresentaram múltiplas comorbidades, sendo a média observada de $3,2 \pm 1,4$ por paciente. A hipertensão arterial foi a comorbidade mais prevalente, com predomínio em $49,1 \%$ dos pacientes, seguida, respectivamente, de doença cardíaca não especificada (42,3\%). Pode-se observar que o uso de fraldas estava presente em $91,5 \%$ dos pacientes e que mais da metade destes eram restritos ao leito $(64,4 \%)$. Dentre o total de 59 pacientes, 14 (23,7\%) apresentaram ITU recorrente, com média de idade de 83,5 \pm 10,8 e taxa de 5,5 episódios/paciente (Tabela 1). 
Tabela 1 - Características clínicas e demográficas da população estudada.

\begin{tabular}{ll}
\hline Características & $\mathrm{n}=59$ \\
\hline Idade (anos), média $\pm \mathrm{dp}$ & $84,4 \pm 9,5$ \\
\hline Gênero, $\mathbf{n}(\%)$ & \\
\hline Feminino & $49(83,1)$ \\
Masculino & $10(16,9)$ \\
\hline Infecção de repetição, $\mathbf{n}(\%)$ & $14(23,7)$ \\
\hline Comorbidades, $\mathbf{n}(\%)$ & \\
\hline Hipertensão arterial & $29(49,1)$ \\
Doença Cardíaca & $25(42,3)$ \\
Doença de Alzheimer & $17(28,8)$ \\
Diabetes Melito tipo 2 & $14(23,7)$ \\
Doença Pulmonar & $11(18,6)$ \\
Demência não especificada & $8(13,5)$ \\
Doença de Parkinson & $6(10,1)$ \\
Infarto Agudo do Miocárdio & $3(5)$ \\
Número de comorbidades, média \pm dp & $3,2 \pm 1,4$ \\
\hline Autonomia, $\mathbf{n}$ (\%) & \\
\hline Uso de fraldas & $54(91,5)$ \\
Restrição ao leito & $38(64,4)$ \\
\hline Legenda: n: número de pacientes; dp: desvio padrão. Fonte: Silva LR, et al., 2020.
\end{tabular}

Dentre os sintomas encontrados houve: baixa prevalência de disúria (6\%); febre $(21,8 \%)$ e a elevada alteração no nível de consciência $(95,4 \%)$ nesta população idosa.

O agente etiológico mais frequente foi a Escherichia coli ( $n=66)$, apresentando mais que o dobro da taxa de isolamento que a Klebsiella sp ( $\mathrm{n}=32$ ). O Enterococcus sp foi isolado em 11 amostras (8,3\%). Observa-se que esse predomínio de frequência da Escherichia coli se mantém inalterado mesmo quando se classifica a ITU em episódio único ou recorrente. A Klebsiella sp também se mantém em segundo lugar, e a seguir, com frequência bem menor, a Pseudomonas aeruginosa nas ITU de repetição e o Enterococcus sp nos pacientes com episódio único (Gráfico 1).

Gráfico 1 - Frequência dos agentes etiológicos isolados nas uroculturas dos pacientes com ITU de repetição e dos que apresentaram episódio único.

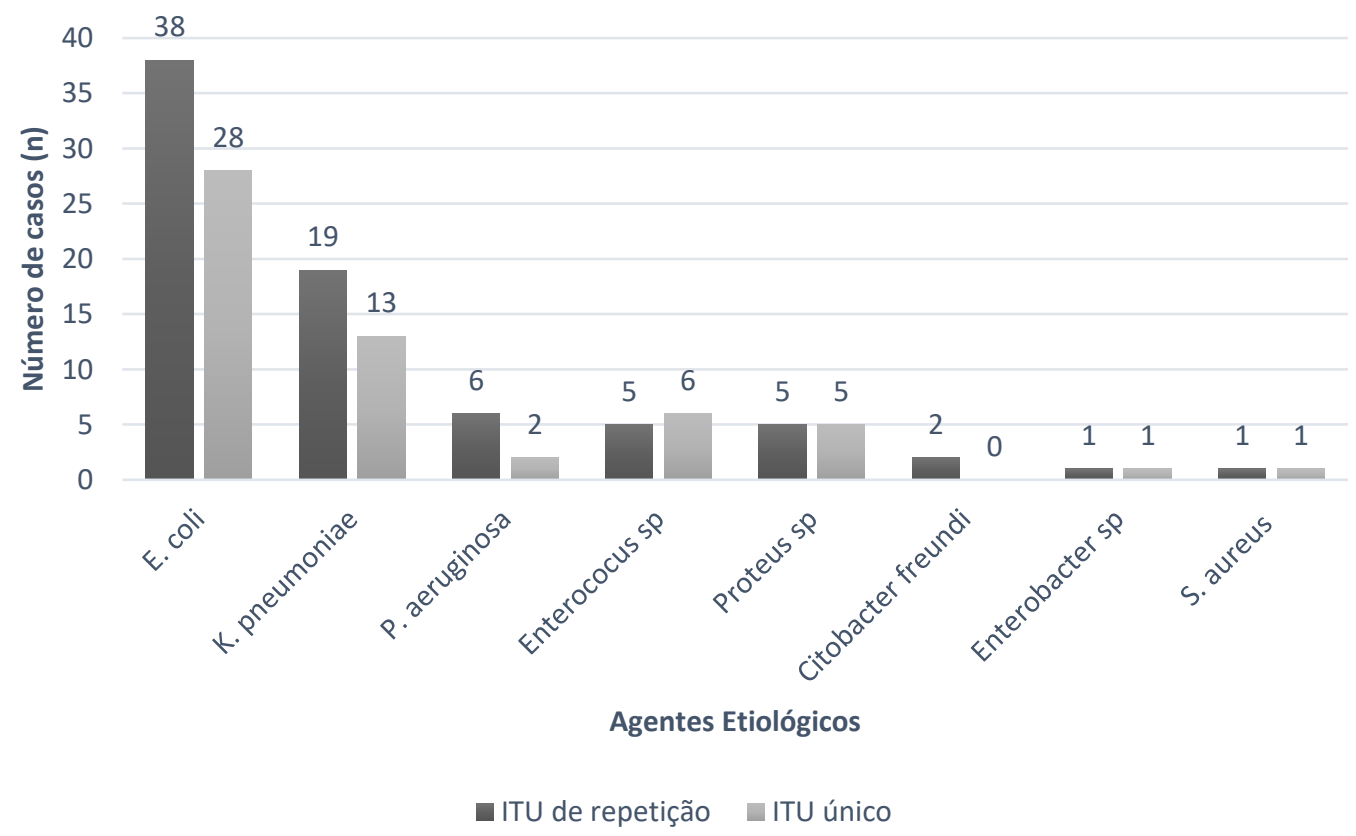

Fonte: Silva LR, et al., 2020. 
Ao analisar o perfil de sensibilidade, respectivamente, para os 02 principais agentes E. coli e Klebsisiela $s p$ para ITU não recorrente nesta população, observou-se baixa sensibilidade para sulfametoxazol + trimetoprim (53,5\% e 46,1\%) e para o ciprofloxacino (53,5\% e 38,4\%). Chama atenção a discrepância na sensibilidade para Ceftriaxona (E. coli: $75 \%$ e Klebisiela:30\%) e para a Fosfomicina (E. coli: $96,4 \%$ e Klebisiela: 61,5\%) (Gráfico 2).

Gráfico 2 - Perfil de sensibilidade de uropatógenos em ITU não recorrentes.

= E.Coli $=$ Klebisiela sp

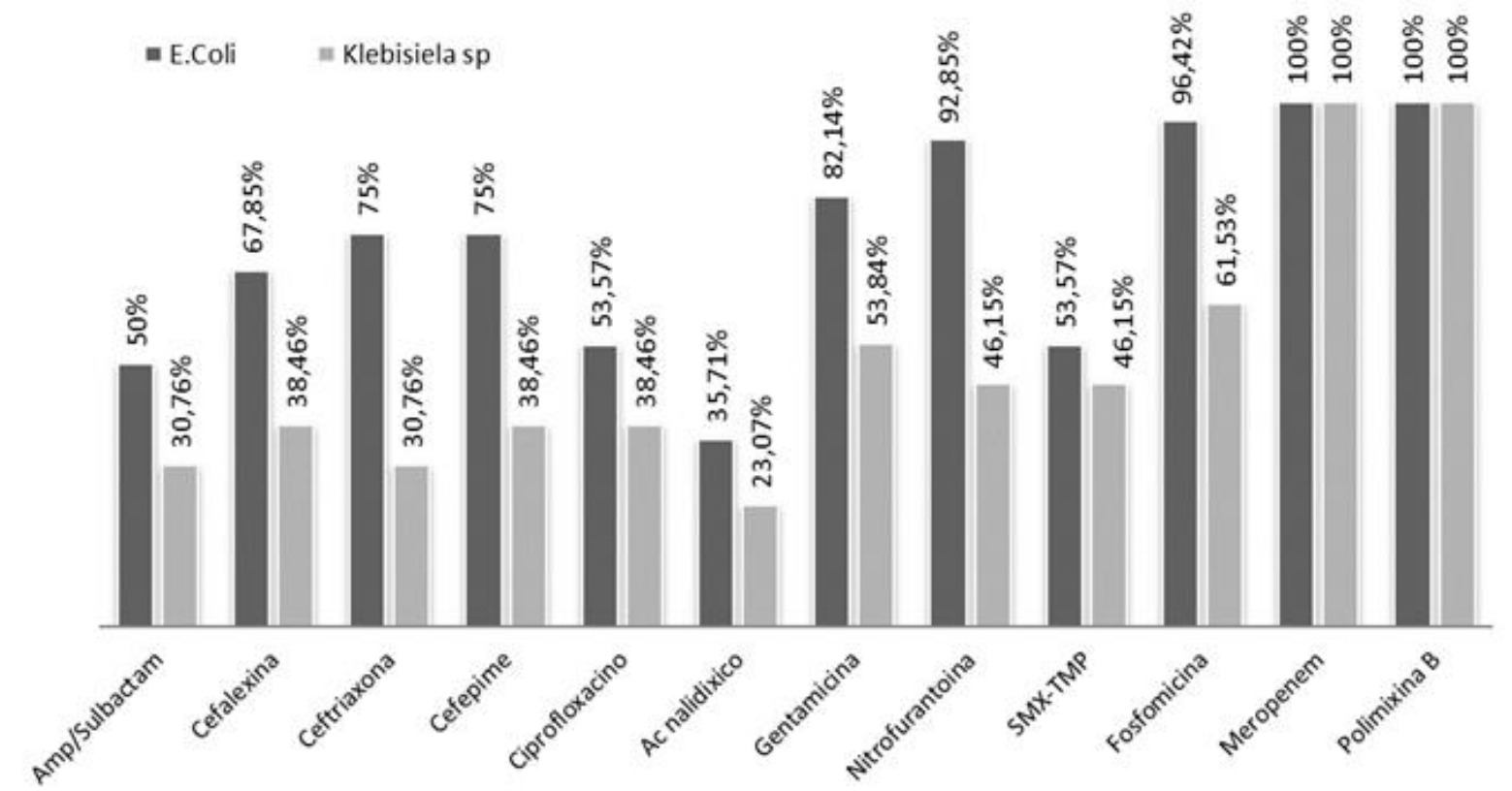

Fonte: Silva LR, et al., 2020.

Por outro lado, quando se analisa o perfil de sensibilidade para ITU de repetição para os agentes $E$. coli e Klebsisiela $s p$ observou-se nesta população baixa sensibilidade para sulfametoxazol + trimetoprim (50\% e $44,4 \%$ ) e para o ciprofloxacino (50\% e 27,7\%) (Gráfico 3).

Gráfico 3 - Perfil de sensibilidade de uropatógenos em ITU de repetição.

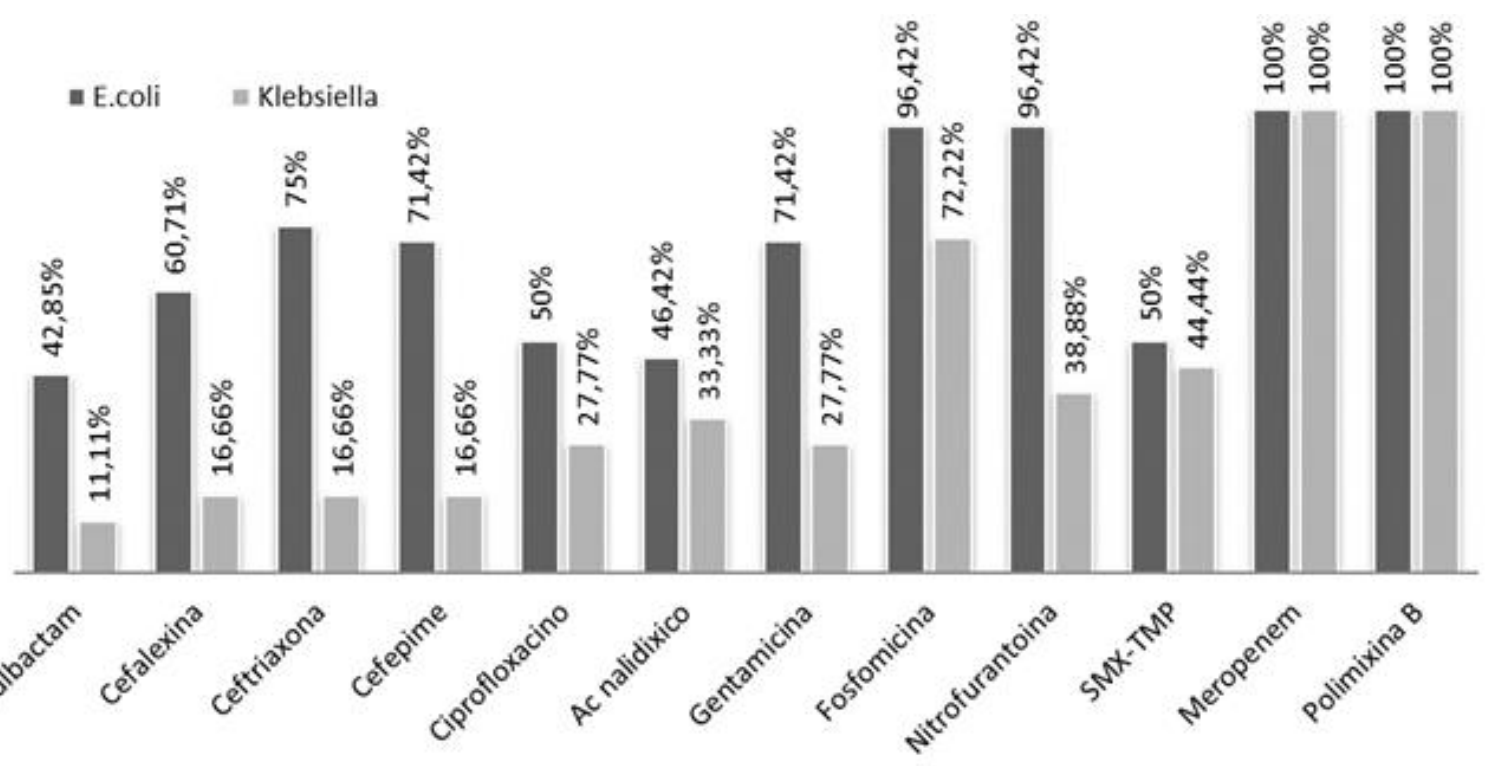

Fonte: Silva LR, et al., 2020. 
Ao se analisar as características clínicas da população e a ocorrência de ITU de repetição, observou-se que as variáveis idade, gênero, HAS, DM, presença de cardiopatia e uso de fralda não se associaram com ITU de repetição.

Por outro lado, a presença de Doença de Alzheimer $(0,28 ; \mathrm{IC}-95 \% 0,02-0,5 ; p=0,01)$ e a restrição ao leito $(0,43 ;$ IC-95\% $0,19-0,6 ; p=0,0003)$ apresentaram correlação significativa com a ITU de repetição. A correlação entre Doença de Alzheimer e ITU de repetição foi significantemente forte $(p=1,37)$ apresentandose como um fator de risco para a recorrência da ITU (Tabela 2).

Tabela 2 - Correlação entre pacientes com Doença de Alzheimer e ITU de repetição.

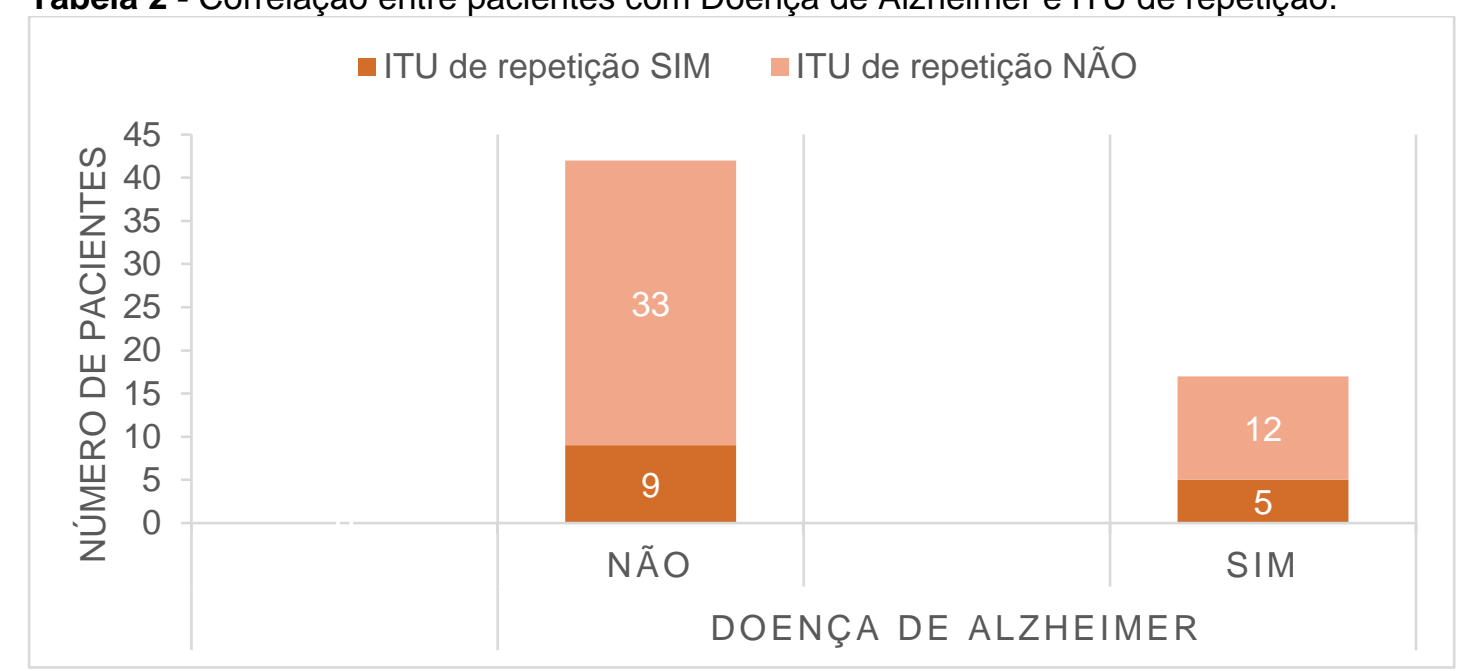

Fonte: Silva LR, et al., 2020.

Outro achado relevante, foi que nos pacientes com ITU, a presença de alteração da característica da urina aumenta significativamente mais com o avançar da idade (0,23; IC-95\% $-0,02-0,47 ; p=0,03)$. Ademais, foi encontrada correlação significativa entre pacientes que apresentaram ITU e possuem Doença de Parkinson com a restrição ao leito $(0,23 ; \mathrm{IC}-95 \%-0,02-0,4 ; \mathrm{IC}: 0,03)$.

\section{DISCUSSÃO}

Os resultados deste estudo confirmam os dados prévios da literatura científica e evidenciam a maior frequência de ITU no sexo feminino. Com o envelhecimento de determinada população ocorre um aumento na prevalência de ITU em ambos os sexos, provavelmente decorrente da diminuição dos mecanismos de defesa do trato urinário. Acredita-se, porém, que isso ocorra de modo mais significativo em idosos do gênero masculino. Nesta pesquisa não houve correlação estatística entre gênero e infecção urinária de repetição. (BEVERIDGE LA, et al., 2011; MODY L e JUTHANI-MEHTA M, 2014).

Em relação à incidência de sintomas associados à infecção do trato urinário em pacientes institucionalizados em lares para idosos, existem divergências na literatura científica. Um estudo mostra que os principais sintomas são alteração do estado mental, febre e disúria, respectivamente. Em outra publicação, a alteração no estado mental e nas características da urina, são os sintomas de maior ocorrência. No presente estudo foi observado que alteração nas características da urina (hematúria, alteração na cor e odor da urina) e a alteração no estado mental foram os sintomas mais prevalentes e os idosos mais velhos apresentam significativamente mais eventos de alteração das características urinárias. (BEVERIDGE LA, et al., 2011; JUTHANI-MEHTA M, et al., 2009).

Nesta pesquisa não houve correlação significante entre DM tipo 2 e ITU, o que diverge da maior parte da literatura científica. Pacientes portadores de DM apresentam maior prevalência de ITU, devido aos vários fatores potenciais, tais como a glicosúria, atividade granulocítica alterada e microangiopatia;baixo clearence microbiano durante a miç̧ão, baixa penetração dos antimicrobianos e do esvaziamento incompleto vesical 
devido à neuropatia. Neste estudo, o baixo número de prontuários analisados pode justificar tal divergência. (NITZAN O, et al., 2015; GEERLINGS SE, 2008).

Doenças crônicas neurodegenerativas, como Doença de Alzheimer e Doença de Parkinson estão associados à bexiga neurogênica. Estas condições causam esvaziamento incompleto da bexiga e refluxo ureteral, que contribuem para a alta frequência de bacteriúria. Isto corrobora com a correlação significativa da Doença de Alzheimer com a ITU de repetição neste estudo, porém não com a Doença de Pakinson, já que esta não teve correlação significativa. (BEVERIDGE LA, et al., 2011; D'AGATA E, et al., 2013; PINHEIRO JES, 2013).

Neste estudo houve relação estatística significativa $(p<0,05)$ entre restrição ao leito com a doença de Parkinson. A literatura relata que a doença de Parkinson é uma das patologias que leva o idoso à imobilidade. Quando esta se torna prolongada, leva à deterioração funcional progressiva de vários sistemas, incluindo o sistema urinário, contribuindo para a incidência de $40 \%$ de casos de ITU nesses pacientes. (PINHEIRO JES, 2013; LEDUC MMS, 2013; ROGERS MAM, et al., 2008).

Assim como outros estudos, nossos achados demonstram que a Escherichia coli é o agente etiológico mais prevalente, seguida da Klebsiella $s p$ e das Gram-positivas Enterococcus sp. A E. coli possui características peculiares que facilitam a ITU como a presença de antígenos que lhe conferem mobilidade; capacidade de interferir na opsonização e fagocitose, além de diminuir o peristaltismo da musculatura lisa do ureter, o que facilita a adesão e portanto, a eliminação pelo fluxo urinário. (NICOLLE LE, 2009).

Estudos mostram que $50-70 \%$ dos indivíduos portadores de ITU de repetição terão resultado de urocultura positiva de 4 a 6 semanas após terapia antimicrobiana. Este resultado nem sempre está associado a sintomas. É importante ressaltar que na ausência de sintomas o tratamento medicamentoso não é indicado mesmo se a cultura for positiva. $O$ objetivo nesta população é aliviar os sintomas e não esterilizar a urina. (NICOLLE LE e YOSHIKAWA TT, 2000).

As bactérias que causam ITU no idoso são, em geral, mais resistentes que na população mais jovem, porque a maior frequência desses processos, habitualmente, gera necessidade de repetidos ciclos de antibióticos predispondo a seleção de cepas com maior resistência. É importante ressaltar que a terapia antimicrobiana, para estes microorganismos, deve ser dirigida por urocultura com antibiograma. Muitas vezes, a terapia parenteral pode ser necessária, quando o agente não é mais suscetível às medicações orais. (TRAJANO HBP e CALDAS CP, 2008; MODY L e JUTHANI-MEHTA M, 2014).

A sensibilidade aos antimicrobianos diminui nos episódios de repetição, em relação aos uropatógenos analisados Escherichia coli e Klebsiella sp. Aminoglicosídeos, quinolonas e associações como SMX-TMP, por exemplo, são menos sensíveis aos germes nas infecções de repetição. O fármaco de escolha nos episódios de ITU de repetição é a Ceftriaxona, administrada via endovenosa. (TRAJANO HBP e CALDAS CP, 2008).

Estudos envolvendo antibioticoterapia profilática, com enfoque nas fluoroquinolonas, beta-lactâmicos e nitrofurantoína demonstraram algum benefício na redução de ITU de repetição em mulheres e homens em comparação ao uso do placebo. O medicamento, horário, dose ou duração ideais para esta profilaxia ainda não foram determinados. (MATTHEWS SJ e LANCASTER JW, 2011).

O uso de Cranberry (Vaccinium macrocarpon), em cápsulas de suco ou extrato concentrado, tem sido o agente mais promissor na redução da recorrência de ITU na população idosa. Seu principal mecanismo de ação é explicado pela presença de proantocianidina em sua composição que inibe a adesão bacteriana às células epiteliais; outra característica do Cranberry é neutralizar o pH, reduzindo a capacidade de adesão bacteriana. Outra terapia profilática indicada seria o uso do estrogênio tópico em mulheres na pósmenopausa, seu benefício está relacionado às alterações vaginais, com consequente diminuição na taxa de colonização vaginal e prevenção de reinfecção do trato urinário. (MATTHEWS SJ e LANCASTER JW, 2011; FRANÇA ACYR, et al., 2014; HEILBERG IP e SCHOR N, 2008).

O número de prontuários analisados é uma das limitações deste estudo, bem como a escassez na literatura a respeito da infecção do trato urinário em pacientes idosos sendo atendidos em seus domicílios. 


\section{CONCLUSÃO}

O presente estudo demonstra que nos pacientes idosos atendidos em domicílio provenientes de serviço de saúde suplementar, os episódios de ITU apresentaram maior incidência do sexo feminino e as manifestações clínicas mais significativas foram a alteração nas características da urina e a alteração do estado mental. A Escherichia coli e a Klebsiella sp foram os uropatógenos mais frequentes isolados em uroculturas. $O$ estudo contribui para pesquisas futuras sobre tratamento domiciliar de idosos com infecção do trato urinário, como também para realizar o diagnóstico precoce baseado nas manifestações clínicas mais comuns dessa população específica. A antibioticoterapia deve ser dirigida de acordo com o perfil de suscetibilidade do uropatógeno, considerando possíveis efeitos adversos aos antibióticos, outros medicamentos usados concomitantemente e as comorbidades do paciente.

\section{REFERÊNCIAS}

1. BEVERIDGE LA, et al. Optimal management of urinary tract infections in older people. Clinical Interventions in Aging, 2011; 6: 173-180.

2. CORRÊA EF, MONTALVÃO ER. Infecção do trato urinário em geriatria. Estudos, 2010; 37(7/8): 625-635.

3. D'AGATA E, et al. Challenges in assessing nursing home residents with advanced dementia for suspected urinary tract infection. Journal of the American Geriatrics Society, 2013; 61(1): 62-66.

4. FRANÇA ACYR, et al. O consumo do cranberry no tratamento de doenças inflamatórias. Ensaios e Ciência: Ciências Biológicas, Agrárias e da Saúde, 2014; 18(1): 47-53.

5. GEERLINGS SE. Urinary tract infections in patients with diabetes mellitus: epidemiology, pathogenesis and treatment. International Journal of Antimicrobial Agents, 2008; 31(1): 54-57.

6. GUPTA K, et al. Acute uncomplicated cystitis and pyelonephritis in women. Clinical Infectious Diseases, 2011; 52(5): 103-120.

7. HEILBERG IP, SCHOR N. Abordagem diagnóstica e terapêutica na infecção do trato urinário - Itu. Revista da Associação Médica Brasileira, 2003; 49(1): 109-116.

8. JUTHANI-MEHTA M, et al. Clinical features to identify urinary tract infection in nursing home residents: a cohort study. Journal of the American Geriatrics Society, 2009; 57(6): 963-970.

9. LEDUC MMS. Imobilidade e síndrome da imobilização. Tratado de geriatria e gerontologia. 3rd. ed. Rio de Janeiro: Guanabara Koogan, 2013; 1546-1561p.

10. MATTHEWS SJ, LANCASTER JW. Urinary tract infections in the elderly population. The American Journal of Geriatric Pharmacotherapy, 2011; 9(5), 286-309.

11. MODY L, JUTHANI-MEHTA M. Urinary tract infections in older women: a clinical review. Journal of the American Medical Association, 2014; 311(8): 844-854.

12. NICOLLE LE. Urinary Tract Infection in the Elderly. Clinics in Geriatric Medicine, 2009; 25: 423-436.

13. NICOLLE LE, YOSHIKAWA TT. Urinary tract infection in long-term-care facility residents. Clinical Infectious Diseases, 2000; 31(3):757-761.

14. NITAZN O, et al. Urinary tract infections in patients with type 2 diabetes mellitus: review of prevalence, diagnosis, and management. Diabetes Metabolic Syndrome and Obesity: Targets and Therapy, 2015; 8: 129-136.

15. PINHEIRO JES. Doença de Parkinson e outros transtornos do movimento. Tratado de geriatria e gerontologia. 3rd. ed. Rio de Janeiro: Guanabara Koogan, 2013; 437-445p.

16. ROGERS MAM, et al. Mobility and other predictors of hospitalization for urinary tract infection: a retrospective cohort study. BMC Geriatrics, 2008; 8: 31.

17. RORIZ-FILHO JS, et al. Infecção do trato urinário. Medicina (Ribeirão Preto), 2010; 43(2):118-125.

18. TRAJANO HBP, CALDAS CP. Uso de antibióticos em idosos hospitalizados com infecção do trato urinário. Revista Hospital Universitário Pedro Ernesto, 2008; 7(1): 116-126.

19. VERAS R. Envelhecimento populacional contemporâneo: demandas, desafios e inovações. Revista de Saúde Pública, 2009; 43(3): 548-54. 